\title{
Global Asymptotic Stability for Linear Fractional Difference Equation
}

\author{
A. Brett, E. J. Janowski, and M. R. S. Kulenović \\ Department of Mathematics, University of Rhode Island, Kingston, RI 02881-0816, USA \\ Correspondence should be addressed to M. R. S. Kulenović; kulenm@math.uri.edu
}

Received 17 March 2014; Accepted 11 May 2014; Published 1 July 2014

Academic Editor: Zong-Xuan Chen

Copyright (C) 2014 A. Brett et al. This is an open access article distributed under the Creative Commons Attribution License, which permits unrestricted use, distribution, and reproduction in any medium, provided the original work is properly cited.

Consider the difference equation $x_{n+1}=\left(\alpha+\sum_{i=0}^{k} a_{i} x_{n-i}\right) /\left(\beta+\sum_{i=0}^{k} b_{i} x_{n-i}\right), n=0,1, \ldots$, where all parameters $\alpha, \beta, a_{i}, b_{i}, i=0,1, \ldots, k$, and the initial conditions $x_{i}, i \in\{-k, \ldots, 0\}$ are nonnegative real numbers. We investigate the asymptotic behavior of the solutions of the considered equation. We give easy-to-check conditions for the global stability and global asymptotic stability of the zero or positive equilibrium of this equation.

\section{Introduction}

Consider the difference equation

$$
x_{n+1}=\frac{\alpha+\sum_{i=0}^{k} a_{i} x_{n-i}}{\beta+\sum_{i=0}^{k} b_{i} x_{n-i}}, \quad n=0,1, \ldots,
$$

where $k \in\{1, \ldots\}$, the parameters $\alpha, \beta, a_{i}, b_{i}, i=0,1, \ldots, k$, and the initial conditions $x_{i}, i \in\{-k, \ldots, 0\}$ are nonnegative real numbers. The important special cases of (1) are the wellknown Riccati equation

$$
x_{n+1}=\frac{\alpha+a_{0} x_{n}}{\beta+b_{0} x_{n}}, \quad n=0,1, \ldots,
$$

the second order linear fractional difference equation

$$
x_{n+1}=\frac{\alpha+\sum_{i=0}^{1} a_{i} x_{n-i}}{\beta+\sum_{i=0}^{1} b_{i} x_{n-i}}, \quad n=0,1, \ldots,
$$

and the third order linear fractional difference equation that we get from (1) for $k=2$. The global behavior and the exact solutions of (2) even for real parameters have been found in [1]. The global behavior of solutions of (3), in many subcases when one or more parameters are zero, was established in [1]. There are still some conjectures left whose answers will complete the global picture of the asymptotic behavior for the solutions of (3). As far as the third order linear fractional difference equation is concerned, there are a large number of sporadic results that are systemized in a book [2]. The characterization of the global asymptotic behavior of the solutions of (1) for $k=2$ seems to be much harder than for the second order equation (3). Consequently an attempt at giving the characterization of the global asymptotic behavior for the solutions of (1) seems to be a formidable task at this time. However using some known global attractivity results we can describe the global asymptotic behavior for the solutions of (1) in some subspaces of the parametric space and the space of initial conditions. See [2-6] for a complete description of the behavior of some special cases of (1), in particular for the cases known as periodic trichotomies. See [7] where the difference in global behavior between the second and third order linear fractional difference equation is emphasized. The results on the global periodicity, that is, the results which describe all special cases of (1) where all solutions are periodic of the same period, were obtained in $[8,9]$. Most results in $[2-6,10,11]$ are based on known global attractivity or global asymptotic stability results obtained in $[1,2,12-17]$.

This paper is an attempt at establishing some global stability results for the equilibrium solution(s) of (1). Our results give effective conditions for global asymptotic stability of the equilibrium solution(s) of (1) expressed in terms of the inequalities on the coefficients. It is worth mentioning that the long standing conjecture for (3) is that local asymptotic stability implies global asymptotic stability of the equilibrium 
$[2,4]$. In the case of the third order equation (1) with $k=2$, the standing conjecture is that local asymptotic stability and boundedness of all solutions imply global asymptotic stability of the equilibrium. If the second conjecture is proved to hold, it will still be very difficult to verify the conditions for local asymptotic stability of the equilibrium as these conditions are very difficult to check for linear fractional equations of order higher than 2. See [2] for many special cases of third order linear fractional equation with very complicated conditions for local asymptotic stability. Thus the presented results are of importance even if the abovementioned conjecture is proved to be true.

The following general global results will be applied to (1); see [18]. Consider the difference equation

$$
x_{n+1}=f\left(x_{n}, \ldots, x_{n-k}\right), \quad n=0,1, \ldots,
$$

where $k \in\{0,1, \ldots\}$. Sometimes it is more advantageous to investigate (4) by embedding (4) into a higher iteration of the form

$$
x_{n+j}=F_{j}\left(x_{n+j-1}, \ldots, x_{n-k}\right), \quad n=0,1, \ldots,
$$

where $j \in\{2,3, \ldots\}$ (see $[16,18,19])$ and then linearizing (4) or (5) by rewriting them (see [18]) into a nonautonomous linear equation of the form

$$
x_{n+l}=\sum_{i=1-l}^{k} g_{i} x_{n-i}, \quad n=0,1, \ldots,
$$

where $l \in\{1,2, \ldots\}$ and the functions $g_{i}: \mathbb{R}^{k+l} \rightarrow \mathbb{R}$ are in general functions of both $n$ and the state variables $x_{i}, i=n-$ $k, \ldots, n+\ell-1$. See $[18,20]$ for examples of such linearizations.

Theorem 1. Let $l \in\{1,2, \ldots\}$. Suppose that (4) has the linearization (6) where the functions $g_{i}: \mathbb{R}^{k+l} \rightarrow \mathbb{R}$ are such that

$$
\sum_{i=1-l}^{k}\left|g_{i}\right| \leq a<1, \quad n=0,1, \ldots
$$

Then

$$
\lim _{n \rightarrow \infty} x_{n}=0
$$

As we have observed in [18], condition (7) is actually a contraction condition in the Banach contraction principle.

In addition, we will need the following stability result which is a consequence of our results in [18].

Theorem 2. Suppose that (4) can be linearized into the form

$$
x_{n+1}-\bar{x}=\sum_{i=0}^{k} g_{i}\left(x_{n-i}-\bar{x}\right), \quad n=0,1, \ldots,
$$

where $\bar{x}$ is an equilibrium of (4) and the functions $g_{i}: \mathbb{R}^{k+1} \rightarrow$ $\mathbb{R}$. If $\sum_{i=0}^{k}\left|g_{i}\right| \leq 1, n \geq 0$, then the equilibrium $\bar{x}$ of (4) is stable.
Proof. Observe that

$$
\left|x_{n+1}-\bar{x}\right| \leq \sum_{i=0}^{k}\left|g_{i}\right|\left|x_{n-i}-\bar{x}\right|, \quad n=0,1, \ldots
$$

Assume that $\sum_{i=0}^{k}\left|x_{-i}-\bar{x}\right|<\delta$. Take $\varepsilon=\delta$. Then (9) implies

$$
\begin{aligned}
& \left|x_{1}-\bar{x}\right| \leq \sum_{i=0}^{k}\left|g_{i}\right|\left|x_{-i}-\bar{x}\right|<\delta \sum_{i=0}^{k}\left|g_{i}\right| \leq \delta, \\
& \left|x_{2}-\bar{x}\right| \leq \sum_{i=0}^{k}\left|g_{i}\right|\left|x_{1-i}-\bar{x}\right|<\delta \sum_{i=0}^{k}\left|g_{i}\right| \leq \delta,
\end{aligned}
$$

and so by induction $\left|x_{n}-\bar{x}\right|<\delta=\varepsilon$ for $n \geq-k$.

\section{Preliminaries}

First observe that when $\sum_{i=0}^{k} b_{i}=0$ (1) becomes the linear nonhomogeneous equation

$$
x_{n+1}=\frac{\alpha}{\beta}+\sum_{i=0}^{k} g_{i} x_{n-i}, \quad n=0,1, \ldots,
$$

where $g_{i}=a_{i} / \beta$ for all $i=0,1, \ldots, k$ and whose equilibrium $\bar{x}$ satisfies $\beta \bar{x}=\alpha+\bar{x} \sum_{i=0}^{k} a_{i}$.

We now establish our first result.

Theorem 3. Let $\beta>0$ and $\sum_{i=0}^{k} b_{i}=0$.

(1) If $\alpha=0$ and $\beta>\sum_{i=0}^{k} a_{i}$, then the zero equilibrium of (1) is globally asymptotically stable.

(2) If $\alpha=0$ and $\beta=\sum_{i=0}^{k} a_{i}$, then the zero equilibrium of (1) is stable.

(3) If $\alpha=0$ and $\beta<\sum_{i=0}^{k} a_{i}$, then $\lim _{n \rightarrow \infty} x_{n}=\infty$ whenever, for some $n \geq 0, x_{n-i}>0, i=0, \ldots, k$.

(4) If $\alpha>0$ and $\beta>\sum_{i=0}^{k} a_{i}$, then the unique positive equilibrium of (1) is globally asymptotically stable.

Proof. When $\alpha=0$ and $\sum_{i=0}^{k} b_{i}=0$, (1) becomes

$$
x_{n+1}=\sum_{i=0}^{k} \frac{a_{i}}{\beta} x_{n-i}, \quad n=0,1, \ldots
$$

(1) In this case $\sum_{i=0}^{k}\left|g_{i}\right|=\sum_{i=0}^{k}\left(a_{i} / \beta\right)<1$ and the result follows from Theorems 1 and 2 .

(2) In this case $\sum_{i=0}^{k}\left|g_{i}\right|=\sum_{i=0}^{k}\left(a_{i} / \beta\right)=1$ and the result follows from Theorem 2 .

(3) Since $g_{i} \geq 0, i \in\{0, \ldots, k\}$ and $\sum_{i=0}^{k} g_{i}=$ $\sum_{i=0}^{k}\left(a_{i} / \beta\right)>1$, then the result follows from Theorem 2 in [18].

When $\alpha>0$ and $\sum_{i=0}^{k} b_{i}=0$, (1) becomes

$$
x_{n+1}=\frac{\alpha}{\beta}+\sum_{i=0}^{k} \frac{a_{i}}{\beta} x_{n-i}, \quad n=0,1, \ldots,
$$


and has a unique positive equilibrium $\bar{x}$ provided $\beta>\sum_{i=0}^{k} a_{i}$. Then

$$
\begin{aligned}
x_{n+1}-\bar{x} & =\sum_{i=0}^{k} \frac{a_{i}}{\beta}\left(x_{n-i}-\bar{x}\right)+\frac{\bar{x} \sum_{i=0}^{k} a_{i}+\alpha-\beta \bar{x}}{\beta} \\
& =\sum_{i=0}^{k} \frac{a_{i}}{\beta}\left(x_{n-i}-\bar{x}\right) .
\end{aligned}
$$

Let $y_{n}=x_{n}-\bar{x}$ where $n \geq 0, \bar{x}>0$. Then, for $n \geq 0, y_{n}$ satisfies

$$
y_{n+1}=\sum_{i=0}^{k} \frac{a_{i}}{\beta} y_{n-i}
$$

(4) Since $\sum_{i=0}^{k}\left|g_{i}\right|=\sum_{i=0}^{k}\left(a_{i} / \beta\right)<1$, then, by Theorem 1 , $\lim _{n \rightarrow \infty} y_{n}=0$ and so $\lim _{n \rightarrow \infty} x_{n}=\bar{x}$. Thus $\bar{x}$ is a global attractor. By applying Theorem 2 to (15) we get that the equilibrium $\bar{x}$ is stable and so the positive equilibrium of (1) is globally asymptotically stable.

For the remainder of this paper we will assume that $b_{i}>0$ for at least one $i \in\{0, \ldots, k\}$.

Now we investigate the stability of the zero equilibrium of (1). Note that (1) has a zero equilibrium if and only if $\alpha=0$ and $\beta \neq 0$.

Theorem 4. Let $\beta>0$ and $\sum_{i=0}^{k} b_{i}>0$. Assume that $\alpha=0$.

(1) If $\beta>\sum_{i=0}^{k} a_{i}>0$, then the zero equilibrium of (1) is globally asymptotically stable.

(2) If $\beta=\sum_{i=0}^{k} a_{i}>0$, then the zero equilibrium of (1) is stable.

Proof. When $\alpha=0$ (1) becomes

$$
x_{n+1}=\frac{\sum_{i=0}^{k} a_{i} x_{n-i}}{\beta+\sum_{i=0}^{k} b_{i} x_{n-i}}, \quad n=0,1, \ldots
$$

which can be written in the linearized form (6) where $l=1$ :

$$
x_{n+1}=\sum_{i=0}^{k} \frac{a_{i}}{\beta+\sum_{i=0}^{k} b_{i} x_{n-i}} x_{n-i}, \quad n=0,1, \ldots
$$

Define $h_{i}=a_{i} /\left(\beta+\sum_{i=0}^{k} b_{i} x_{n-i}\right), n \geq 0$ for $i=0, \ldots, k$.

(1) The proof follows from Theorems 1 and 2 as $\sum_{i=0}^{k}\left|h_{i}\right|=$ $\sum_{i=0}^{k}\left(a_{i} /\left(\beta+\sum_{i=0}^{k} b_{i} x_{n-i}\right)\right) \leq \sum_{i=0}^{k} a_{i} / \beta<1$.

(2) The proof follows from Theorem 2 as $\sum_{i=0}^{k}\left|h_{i}\right|=$ $\sum_{i=0}^{k}\left(a_{i} /\left(\beta+\sum_{i=0}^{k} b_{i} x_{n-i}\right)\right) \leq \sum_{i=0}^{k} a_{i} / \beta=1$.

\section{Positive Equilibrium}

In this section we investigate the stability of the unique positive equilibrium of (1) by using Theorems 1 and 2 .

Note that, for $n=0,1, \ldots$, the function

$$
f\left(x_{n}, \ldots, x_{n-k}\right)=\frac{\alpha+\sum_{i=0}^{k} a_{i} x_{n-i}}{\beta+\sum_{i=0}^{k} b_{i} x_{n-i}}
$$

has the following properties:

(a) if $a_{i}>0$ and $b_{i}=0$, then $f$ is increasing in $x_{n-i}$ on the interval $[0, \infty)$;

(b) if $a_{i}=0$ and $b_{i}>0$, then $f$ is decreasing in $x_{n-i}$ on the interval $[0, \infty)$.

The following result gives some other cases when $f$ is monotonic.

Remark 5. Consider the function $f$ given by $(19)$ on $[0, \infty)^{k+1}$ where $\bar{x}$ is a unique positive fixed point of this function. Assume that $a_{i}, b_{i}>0$ for $i \in\{0, \ldots, k\}$. Then for $k \in\{0,1, \ldots\}$ set

$$
f\left(u_{0}, \ldots, u_{k}\right)=\frac{\alpha+\sum_{i=0}^{k} a_{i} u_{i}}{\beta+\sum_{i=0}^{k} b_{i} u_{i}}, \quad u_{i} \in[0, \infty), i=0, \ldots, k
$$

Then for $i \in\{0, \ldots, k\}$

$$
\begin{aligned}
& f_{u_{i}}=\frac{\partial f}{\partial u_{i}}=\frac{\left(\beta+\sum_{j=0}^{k} b_{j} u_{j}\right) a_{i}-\left(\alpha+\sum_{j=0}^{k} a_{j} u_{j}\right) b_{i}}{\left(\beta+\sum_{j=0}^{k} b_{j} u_{j}\right)^{2}} \\
&=b_{i} \frac{a_{i} / b_{i}-f\left(u_{0}, \ldots, u_{k}\right)}{\beta+\sum_{j=0}^{k} b_{j} u_{j}}, \\
& f_{u_{i}}(\bar{x}, \ldots, \bar{x})=\frac{a_{i}-\bar{x} b_{i}}{\beta+\bar{x} \sum_{j=0}^{k} b_{j}} .
\end{aligned}
$$

Thus we have that, for $i \in\{0, \ldots, k\}, f_{u_{i}}>0$ on the interval $\left[0, a_{i} / b_{i}\right)$ and $f_{u_{i}}<0$ on the interval $\left(a_{i} / b_{i}, \infty\right)$.

In the case when $f$ is monotonic in all its arguments one can try to use global attractivity and global asymptotic stability results established in $[1,2,15,16]$.

In order to apply Theorem 1 to (1) we first need to linearize (1) into the form (6) which can be done as follows:

$$
\begin{aligned}
& x_{n+1}-\bar{x}= \frac{\sum_{i=0}^{k} a_{i}\left(x_{n-i}-\bar{x}\right)}{\beta+\sum_{i=0}^{k} b_{i} x_{n-i}} \\
&+\frac{\alpha+\bar{x} \sum_{i=0}^{k} a_{i}-\beta \bar{x}-\bar{x} \sum_{i=0}^{k} b_{i} x_{n-i}}{\beta+\sum_{i=0}^{k} b_{i} x_{n-i}}, \\
& n=0,1, \ldots .
\end{aligned}
$$


Now applying the equilibrium equation we get that for $n \geq 0$

$$
\begin{aligned}
x_{n+1}-\bar{x} & =\frac{\sum_{i=0}^{k} a_{i}\left(x_{n-i}-\bar{x}\right)}{\beta+\sum_{i=0}^{k} b_{i} x_{n-i}}-\frac{\bar{x} \sum_{i=0}^{k} b_{i}\left(x_{n-i}-\bar{x}\right)}{\beta+\sum_{i=0}^{k} b_{i} x_{n-i}} \\
& =\sum_{i=0}^{k} \frac{a_{i}-\bar{x} b_{i}}{\beta+\sum_{i=0}^{k} b_{i} x_{n-i}}\left(x_{n-i}-\bar{x}\right) .
\end{aligned}
$$

Let $y_{n}=x_{n}-\bar{x}$ for $n \geq 0$ and $\bar{x}>0$. Then $y_{n}$ satisfies

$$
y_{n+1}=\sum_{i=0}^{k} g_{i} y_{n-i}, \quad n=0,1, \ldots
$$

where for $i=0, \ldots, k$

$$
g_{i}=\frac{a_{i}-\bar{x} b_{i}}{\beta+\sum_{i=0}^{k} b_{i} x_{n-i}}, \quad n=0,1, \ldots
$$

The conditions $a_{i}>b_{i} \bar{x}, a_{i}<b_{i} \bar{x}$ and $a_{i}=b_{i} \bar{x}$, which are equivalent to $g_{i}>0, g_{i}<0$, and $g_{i}=0$, can be reformulated in a more explicit way.

Proposition 6. Let $a_{j}, b_{j}>0$ for some $j \in\{0, \ldots, k\}$ and let $\bar{x}$ be the positive equilibrium of (1). Then for $j \in\{0, \ldots, k\}$

(a) $a_{j}>b_{j} \bar{x}$ if and only if $a_{j}^{2} \sum_{i=0}^{k} b_{i}+a_{j} b_{j}\left(\beta-\sum_{i=0}^{k} a_{i}\right)>$ $\alpha b_{j}^{2}$

(b) $a_{j}<b_{j} \bar{x}$ if and only if $a_{j}^{2} \sum_{i=0}^{k} b_{i}+a_{j} b_{j}\left(\beta-\sum_{i=0}^{k} a_{i}\right)<$ $\alpha b_{j}^{2}$

(c) $a_{j}=b_{j} \bar{x}$ if and only if $a_{j}^{2} \sum_{i=0}^{k} b_{i}+a_{j} b_{j}\left(\beta-\sum_{i=0}^{k} a_{i}\right)=$ $\alpha b_{j}^{2}$.

Proof. Consider the following.

Case $1(\alpha=0)$. In this case (1) has the positive equilibrium $\bar{x}=\left(\sum_{i=0}^{k} a_{i}-\beta\right) / \sum_{i=0}^{k} b_{i}$ provided $\sum_{i=0}^{k} a_{i}>\beta$. Now case (a) becomes

$$
a_{j}>\frac{\left(\sum_{i=0}^{k} a_{i}\right)-\beta}{\sum_{i=0}^{k} b_{i}} b_{j}
$$

if and only if $a_{j} \sum_{i=0}^{k} b_{i}>\left(\sum_{i=0}^{k} a_{i}-\beta\right) b_{j}$ which proves (a). The proofs of parts (b) and (c) are similar.

Case $2(\alpha>0)$. Then (1) has the positive equilibrium

$$
\bar{x}=\frac{-\left(\beta-\sum_{i=0}^{k} a_{i}\right)+\sqrt{\left(\beta-\sum_{i=0}^{k} a_{i}\right)^{2}+4 \alpha \sum_{i=0}^{k} b_{i}}}{2 \sum_{i=0}^{k} b_{i}} .
$$

(a) Assume that $a_{j}>b_{j} \bar{x}$. Then

$$
a_{j}>b_{j}\left[\frac{-\left(\beta-\sum_{i=0}^{k} a_{i}\right)+\sqrt{\left(\beta-\sum_{i=0}^{k} a_{i}\right)^{2}+4 \alpha \sum_{i=0}^{k} b_{i}}}{2 \sum_{i=0}^{k} b_{i}}\right]
$$

implies

$$
\begin{aligned}
& {\left[2 a_{j} \sum_{i=0}^{k} b_{i}+b_{j}\left(\beta-\sum_{i=0}^{k} a_{i}\right)\right]^{2}} \\
& >b_{j}^{2}\left[\left(\beta-\sum_{i=0}^{k} a_{i}\right)^{2}+4 \alpha \sum_{i=0}^{k} b_{i}\right]
\end{aligned}
$$

which yields

$$
\begin{aligned}
4 a_{j}^{2}\left(\sum_{i=0}^{k} b_{i}\right)^{2} & +4 a_{j} b_{j}\left(\beta-\sum_{i=0}^{k} a_{i}\right) \sum_{i=0}^{k} b_{i} \\
& +b_{j}^{2}\left(\beta-\sum_{i=0}^{k} a_{i}\right)^{2} \\
> & b_{j}^{2}\left(\beta-\sum_{i=0}^{k} a_{i}\right)^{2}+4 \alpha b_{j}^{2} \sum_{i=0}^{k} b_{i}
\end{aligned}
$$

and so

$$
a_{j}^{2} \sum_{i=0}^{k} b_{i}+a_{j} b_{j}\left(\beta-\sum_{i=0}^{k} a_{i}\right)>\alpha b_{j}^{2} .
$$

Now assume that $a_{j}^{2} \sum_{i=0}^{k} b_{i}+a_{j} b_{j}\left(\beta-\sum_{i=0}^{k} a_{i}\right)>\alpha b_{j}^{2}$. Then

$$
\begin{aligned}
& \left(2 a_{j} \sum_{i=0}^{k} b_{i}+b_{j}\left(\beta-\sum_{i=0}^{k} a_{i}\right)\right)^{2} \\
& \quad>b_{j}^{2}\left(\left(\beta-\sum_{i=0}^{k} a_{i}\right)^{2}+4 \alpha \sum_{i=0}^{k} b_{i}\right)
\end{aligned}
$$

and so

$$
\begin{aligned}
2 a_{j} \sum_{i=0}^{k} b_{i}+b_{j}\left(\beta-\sum_{i=0}^{k} a_{i}\right) \\
>b_{j} \sqrt{\left(\left(\beta-\sum_{i=0}^{k} a_{i}\right)^{2}+4 \alpha \sum_{i=0}^{k} b_{i}\right)} .
\end{aligned}
$$

Otherwise, suppose that

$$
\begin{aligned}
2 a_{j} \sum_{i=0}^{k} b_{i}+b_{j}\left(\beta-\sum_{i=0}^{k} a_{i}\right) \\
\leq b_{j} \sqrt{\left(\beta-\sum_{i=0}^{k} a_{i}\right)^{2}+4 \alpha \sum_{i=0}^{k} b_{i} .}
\end{aligned}
$$

Since

$$
a_{j}\left[2 a_{j} \sum_{i=0}^{k} b_{i}+b_{j}\left(\beta-\sum_{i=0}^{k} a_{i}\right)\right]>\alpha b_{j}^{2}>0,
$$


then

$$
\begin{gathered}
{\left[2 a_{j} \sum_{i=0}^{k} b_{i}+b_{j}\left(\beta-\sum_{i=0}^{k} a_{i}\right)\right]^{2}} \\
\leq\left[2 a_{j} \sum_{i=0}^{k} b_{i}+b_{j}\left(\beta-\sum_{i=0}^{k} a_{i}\right)\right] \\
\times b_{j} \sqrt{\left(\beta-\sum_{i=0}^{k} a_{i}\right)^{2}+4 \alpha \sum_{i=0}^{k} b_{i}} \\
\leq\left[b_{j} \sqrt{\left(\beta-\sum_{i=0}^{k} a_{i}\right)^{2}+4 \alpha \sum_{i=0}^{k} b_{i}}\right]^{2}
\end{gathered}
$$

which is a contradiction.

Therefore,

$$
a_{j}>b_{j}\left[\frac{-\left(\beta-\sum_{i=0}^{k} a_{i}\right)+\sqrt{\left(\beta-\sum_{i=0}^{k} a_{i}\right)^{2}+4 \alpha \sum_{i=0}^{k} b_{i}}}{2 \sum_{i=0}^{k} b_{i}}\right]
$$

and so $a_{j}>\bar{x} b_{j}$.

(b) Similarly we can show that $a_{j} \geq \bar{x} b_{j}$ if and only if $a_{j}^{2} \sum_{i=0}^{k} b_{i}+a_{j} b_{j}\left(\beta-\sum_{i=0}^{k} a_{i}\right) \geq \alpha b_{j}^{2}$ from which the result follows.

(c) Assume that $a_{j}=\bar{x} b_{j}$. Suppose that

$$
a_{j}^{2} \sum_{i=0}^{k} b_{i}+a_{j} b_{j}\left(\beta-\sum_{i=0}^{k} a_{i}\right) \neq \alpha b_{j}^{2} \text {. }
$$

Then either $a_{j}^{2} \sum_{i=0}^{k} b_{i}+a_{j} b_{j}\left(\beta-\sum_{i=0}^{k} a_{i}\right)>\alpha b_{j}^{2}$ or $a_{j}^{2} \sum_{i=0}^{k} b_{i}+$ $a_{j} b_{j}\left(\beta-\sum_{i=0}^{k} a_{i}\right)<\alpha b_{j}^{2}$ and so either $a_{j}>\bar{x} b_{j}$ or $a_{j}<\bar{x} b_{j}$ which are both contradictions. Thus

$$
a_{j}^{2} \sum_{i=0}^{k} b_{i}+a_{j} b_{j}\left(\beta-\sum_{i=0}^{k} a_{i}\right)=\alpha b_{j}^{2} .
$$

Similarly we can show that $a_{j}^{2} \sum_{i=0}^{k} b_{i}+a_{j} b_{j}\left(\beta-\sum_{i=0}^{k} a_{i}\right)=\alpha b_{j}^{2}$ implies $a_{j}=\bar{x} b_{j}$.

We can now obtain easy-to-check conditions which show when the positive equilibrium of (1) is globally asymptotically stable. We will then apply these conditions to various cases of (1).

Theorem 7. Let $\sum_{i=0}^{k} b_{i}>0$. Assume that one of the following holds:

(1) $\sum_{i=0}^{k}\left|a_{i}-\bar{x} b_{i}\right|<\beta$;

(2) there exist $L, N>0$ such that for every solution $\left\{x_{n}\right\}$ of (1) $x_{n} \geq L$ for all $n \geq N$ and $\sum_{i=0}^{k}\left|a_{i}-\bar{x} b_{i}\right|<\beta+$ $L \sum_{i=0}^{k} b_{i}$, where $\beta \geq 0$.
Then the positive equilibrium $\bar{x}$ of (1) is globally asymptotically stable on the interval $[0, \infty)$.

Proof. As we have seen (1) can be written in the form of the linearized equation (24), where the coefficients $g_{i}$ are given as (25).

(1) Observe that for $n \geq 0$

$$
\begin{aligned}
\sum_{i=0}^{k}\left|g_{i}\right| & =\sum_{i=0}^{k} \frac{\left|a_{i}-\bar{x} b_{i}\right|}{\beta+\sum_{i=0}^{k} b_{i} x_{n-i}} \\
& =\frac{\sum_{i=0}^{k}\left|a_{i}-\bar{x} b_{i}\right|}{\beta+\sum_{i=0}^{k} b_{i} x_{n-i}} \leq \frac{\sum_{i=0}^{k}\left|a_{i}-\bar{x} b_{i}\right|}{\beta}<1 .
\end{aligned}
$$

Then by Theorem $1, \lim _{n \rightarrow \infty} y_{n}=0$ and so $\lim _{n \rightarrow \infty} x_{n}=\bar{x}$. Thus $\bar{x}$ is a global attractor on the interval $[0, \infty)$.

From (23) we have that $g_{i}=\left(a_{i}-\bar{x} b_{i}\right) /\left(\beta+\sum_{i=0}^{k} b_{i} x_{n-i}\right)$, for $i=0,1, \ldots, k$. Then

$$
\sum_{i=0}^{k}\left|g_{i}\right|=\sum_{i=0}^{k}\left|\frac{a_{i}-\bar{x} b_{i}}{\beta+\sum_{i=0}^{k} b_{i} x_{n-i}}\right| \leq \sum_{i=0}^{k} \frac{\left|a_{i}-\bar{x} b_{i}\right|}{\beta}<1,
$$

which by Theorem 2 implies that the equilibrium $\bar{x}$ is stable.

(2) Assume that there exist $L, N>0$ such that for every solution $\left\{x_{n}\right\}$ of (1) $x_{n} \geq L$ for all $n \geq N$. Then for $n \geq 0$

$$
\begin{aligned}
\sum_{i=0}^{k}\left|g_{i}\right| & =\sum_{i=0}^{k} \frac{\left|a_{i}-\bar{x} b_{i}\right|}{\beta+\sum_{i=0}^{k} b_{i} x_{n-i}} \\
& =\frac{\sum_{i=0}^{k}\left|a_{i}-\bar{x} b_{i}\right|}{\beta+\sum_{i=0}^{k} b_{i} x_{n-i}} \leq \frac{\sum_{i=0}^{k}\left|a_{i}-\bar{x} b_{i}\right|}{\beta+L \sum_{i=0}^{k} b_{i}}<1
\end{aligned}
$$

and, so by Theorem $1, \bar{x}$ is a global attractor on the interval $[L, \infty)$. Since, by assumption, $[L, \infty)$ is an attracting interval, then $\bar{x}$ is a global attractor on the interval $[0, \infty)$.

By Theorem 2 applied to (23), $\bar{x}$ is stable. Consequently, $\bar{x}$ is globally asymptotically stable on the interval $[0, \infty)$.

Many cases of (1) have some combination of $a_{i}<\bar{x} b_{i}, a_{i}>$ $\bar{x} b_{i}$, and $a_{i}=\bar{x} b_{i}$. In view of this we will adopt the following 
notations where $I_{>}=\left\{i \mid\right.$ such that $\left.a_{i}>\bar{x} b_{i}\right\}, I_{=}=\{i \mid$ such that $\left.a_{i}=\bar{x} b_{i}\right\}$, and $I_{<}=\left\{i \mid\right.$ such that $\left.a_{i}<\bar{x} b_{i}\right\}$ :

$$
\begin{gathered}
A_{S}=\sum_{i \in I_{>}} a_{i}=\text { the sum of all the } a_{i} \text { 's, } \\
B_{S}=\sum_{i \in I_{>}} b_{i}=\text { the sum of all the } b_{i} \text { 's, }
\end{gathered}
$$$$
\text { such that } a_{i}>\bar{x} b_{i} \text {, }
$$$$
A_{N}=\sum_{i \in I_{=}} a_{i}=\text { the sum of all the } a_{i} \text { 's, }
$$$$
B_{N}=\sum_{i \in I_{=}} b_{i}=\text { the sum of all the } b_{i} \text { 's, }
$$

$$
\text { such that } a_{i}=\bar{x} b_{i} \text {, }
$$

$A_{R}=\sum_{i \in I_{<}} a_{i}=$ the sum of all the $a_{i}$ 's,

$B_{R}=\sum_{i \in I_{<}} b_{i}=$ the sum of all the $b_{i}$ 's,

$$
\text { such that } a_{i}<\bar{x} b_{i} \text {. }
$$

Then $A_{S}+A_{N}+A_{R}=\sum_{i=0}^{k} a_{i}$ and $B_{S}+B_{N}+B_{R}=\sum_{i=0}^{k} b_{i}$. Also $A_{S}>\bar{x} B_{S}, A_{N}=\bar{x} B_{N}$, and $A_{R}<\bar{x} B_{R}$.

Before we apply Theorem 7 to various cases of (1) we establish the following useful lemma.

Lemma 8. Let $\bar{x}, \alpha, \beta, \sum_{i=0}^{k} b_{i}>0$ and $L \geq 0$. Then

$$
\begin{array}{r}
\left(2 \beta+L \sum_{i=0}^{k} b_{i}\right)\left(\beta+\sum_{i=0}^{k} a_{i}+L \sum_{i=0}^{k} b_{i}\right)>\alpha \sum_{i=0}^{k} b_{i} \\
\text { iff } \beta+L \sum_{i=0}^{k} b_{i}>\bar{x} \sum_{i=0}^{k} b_{i}-\sum_{i=0}^{k} a_{i},
\end{array}
$$

(b)

$$
\begin{array}{r}
\left(2 \beta+L \sum_{i=0}^{k} b_{i}\right)\left(\beta+\sum_{i=0}^{k} a_{i}+L \sum_{i=0}^{k} b_{i}\right)=\alpha \sum_{i=0}^{k} b_{i} \\
\text { iff } \beta+L \sum_{i=0}^{k} b_{i}=\bar{x} \sum_{i=0}^{k} b_{i}-\sum_{i=0}^{k} a_{i},
\end{array}
$$

(c)

$$
\begin{array}{r}
\left(2 \beta+L \sum_{i=0}^{k} b_{i}\right)\left(\beta+\sum_{i=0}^{k} a_{i}+L \sum_{i=0}^{k} b_{i}\right)<\alpha \sum_{i=0}^{k} b_{i} \\
\text { iff } \beta+L \sum_{i=0}^{k} b_{i}<\bar{x} \sum_{i=0}^{k} b_{i}-\sum_{i=0}^{k} a_{i} .
\end{array}
$$

Proof. Observe that in Proposition 6 for $j \in\{0, \ldots, k\} a_{j}$ and $b_{j}$ are positive real numbers. Thus by Proposition 6 with $a_{j}=$ $\beta+\sum_{i=0}^{k} a_{i}+L \sum_{i=0}^{k} b_{i}$ and $b_{j}=\sum_{i=0}^{k} b_{i}$ we have that

(a)

$$
\left(2 \beta+L \sum_{i=0}^{k} b_{i}\right)\left(\beta+\sum_{i=0}^{k} a_{i}+L \sum_{i=0}^{k} b_{i}\right)>\alpha \sum_{i=0}^{k} b_{i}
$$

if and only if

$$
\begin{aligned}
& \left(\beta+\sum_{i=0}^{k} a_{i}+L \sum_{i=0}^{k} b_{i}\right)^{2} \sum_{i=0}^{k} b_{i}+\left(\beta+\sum_{i=0}^{k} a_{i}+L \sum_{i=0}^{k} b_{i}\right) \\
& \times\left(\beta-\sum_{i=0}^{k} a_{i}\right) \sum_{i=0}^{k} b_{i}>\alpha\left(\sum_{i=0}^{k} b_{i}\right)^{2}
\end{aligned}
$$

if and only if

$$
\beta+\sum_{i=0}^{k} a_{i}+L \sum_{i=0}^{k} b_{i}>\bar{x} \sum_{i=0}^{k} b_{i}
$$

Cases (b) and (c) follow similarly.

Theorem 9. Let $\beta, \sum_{i=0}^{k} b_{i}>0$. Then the positive equilibrium $\bar{x}$ of (1) is globally asymptotically stable on the interval $[0, \infty)$ provided one of the following holds:

(1) $a_{i}=\bar{x} b_{i}$ for all $i \in\{0, \ldots, k\}$;

(2) $a_{i} \geq \bar{x} b_{i}$ for $i \in\{0, \ldots, k\}$ and $\alpha>0$;

(3) $a_{i} \leq \bar{x} b_{i}$ for $i \in\{0, \ldots, k\}$ and $2 \beta^{2}+2 \beta \sum_{i=0}^{k} a_{i}>$ $\alpha \sum_{i=0}^{k} b_{i}>0$;

(4) for some $i, j \in\{0, \ldots, k\}, a_{i}>\bar{x} b_{i}, a_{j}<\bar{x} b_{j}$ and $\alpha / \bar{x}+$ $2 A_{S}-2 \bar{x} B_{S}<2 \beta$, where $\alpha \geq 0$.

Proof. The positive equilibrium $\bar{x}$ of (1) satisfies

$$
\beta-\frac{\alpha}{\bar{x}}=\sum_{i=0}^{k} a_{i}-\bar{x} \sum_{i=0}^{k} b_{i}
$$

(1) Let $a_{i}=\bar{x} b_{i}$ for all $i \in\{0, \ldots, k\}$. Then $\sum_{i=0}^{k} a_{i}=$ $\bar{x} \sum_{i=0}^{k} b_{i}$ and so $\beta=\alpha / \bar{x}$. Then (1) becomes, for $n \geq 0$,

$$
x_{n+1}=\frac{\alpha+\sum_{i=0}^{k} a_{i} x_{n-i}}{\beta+\sum_{i=0}^{k} b_{i} x_{n-i}}=\frac{\beta \bar{x}+\sum_{i=0}^{k} \bar{x} b_{i} x_{n-i}}{\beta+\sum_{i=0}^{k} b_{i} x_{n-i}}=\bar{x} .
$$

(2) Let $a_{i} \geq \bar{x} b_{i}$ for $i \in\{0, \ldots, k\}$. Then for $i \in\{0, \ldots, k\}$ we have $\left|a_{i}-\bar{x} b_{i}\right|=a_{i}-\bar{x} b_{i}$. Thus

$$
\sum_{i=0}^{k}\left|a_{i}-\bar{x} b_{i}\right|=\sum_{i=0}^{k} a_{i}-\bar{x} \sum_{i=0}^{k} b_{i}=\beta-\frac{\alpha}{\bar{x}}<\beta
$$

and the result follows from Theorem 7. 
(3) Let $a_{i} \leq \bar{x} b_{i}$ for $i \in\{0, \ldots, k\}$. Then for $i \in\{0, \ldots, k\}$ we have $\left|a_{i}-\bar{x} b_{i}\right|=\bar{x} b_{i}-a_{i}$. By Lemma 8 with $L=0$

$$
\sum_{i=0}^{k}\left|a_{i}-\bar{x} b_{i}\right|=\bar{x} \sum_{i=0}^{k} b_{i}-\sum_{i=0}^{k} a_{i}<\beta
$$

and the result follows from Theorem 7.

(4) For some $i, j \in\{0, \ldots, k\}, a_{i}>\bar{x} b_{i}$ and $a_{j}<\bar{x} b_{j}$. Then

$$
\begin{aligned}
\sum_{i=0}^{k}\left|a_{i}-\bar{x} b_{i}\right| & \\
= & \left(A_{S}-\bar{x} B_{S}\right)+\left(\bar{x} B_{N}-A_{N}\right)+\left(\bar{x} B_{R}-A_{R}\right) \\
= & \left(A_{S}-A_{N}-A_{R}\right)+\bar{x}\left(B_{R}+B_{N}-B_{S}\right) \\
= & 2 \bar{x} B_{S}-2 A_{S}-\left(A_{R}+A_{N}-A_{S}\right) \\
& +\bar{x}\left(B_{R}+B_{N}-B_{S}\right)-2 \bar{x} B_{S}+2 A_{S} \\
= & \bar{x}\left(B_{R}+B_{N}+B_{S}\right)-\left(A_{R}+A_{N}+A_{S}\right) \\
& +2 A_{S}-2 \bar{x} B_{S} \\
= & \bar{x} \sum_{i=0}^{k} b_{i}-\sum_{i=0}^{k} a_{i}+2 A_{S}-2 \bar{x} B_{S} .
\end{aligned}
$$

Observe that $\bar{x}\left(B_{R}+B_{N}-B_{S}\right)-\left(A_{R}+A_{N}-A_{S}\right) \geq 0$. Since $\alpha / \bar{x}+2 A_{S}-2 \bar{x} B_{S}<2 \beta$, then

$$
\begin{aligned}
\sum_{i=0}^{k}\left|a_{i}-\bar{x} b_{i}\right| & =\bar{x} \sum_{i=0}^{k} b_{i}-\sum_{i=0}^{k} a_{i}+2 A_{S}-2 \bar{x} B_{S} \\
& =\frac{\alpha}{\bar{x}}-\beta+2 A_{S}-2 \bar{x} B_{S}<\beta
\end{aligned}
$$

and so the result follows from Theorem 7.

There are many cases of (1) when we can establish a lower bound for all the solutions of (1).

Remark 10. The results on boundedness of all solutions of (1) are well known; see $[2,19]$. For instance, if for every $i \in$ $\{0, \ldots, k\}$ such that $b_{i}>0$ we have $a_{i}>0$, then the uniform lower bound $L$ for all solutions $\left\{x_{n}\right\}$ of (1), for $n \geq 1$, is

$$
L=\frac{\min \left\{\alpha, a_{i} \mid a_{i}>0\right\}}{\max \left\{\beta, b_{i} \mid b_{i}>0\right\}} .
$$

On the other hand, if for every $i \in\{0, \ldots, k\}$ such that $a_{i}>$ 0 we have $b_{i}>0$, then the uniform lower bound $L$ for all solutions of (1), for $n \geq 1$, is

$$
L=\frac{\min \left\{\alpha, a_{i} \mid a_{i}>0\right\}}{\max \left\{\beta+U \sum_{j, a_{j}=0} b_{j}, b_{i} \mid b_{i}>0\right\}},
$$

where

$$
U=\frac{\max \left\{\alpha, a_{i} \mid a_{i}>0\right\}}{\min \left\{\beta, b_{i} \mid b_{i}>0\right\}}
$$

See Example 19.

The results of Theorem 9 can be extended for those cases of (1) which have a lower bound $L$ for every solution of (1); see Remark 10.

Theorem 11. Let $\sum_{i=0}^{k} b_{i}>0$ and let $L, N>0$ be such that, for $n \geq N, x_{n} \geq L$ for every solution $\left\{x_{n}\right\}$ of (1).

(1) If $\beta>0$ and either

(a) $a_{i} \geq \bar{x} b_{i}$ for $i \in\{0, \ldots, k\}$ and $\alpha=0$;

(b) $a_{i} \leq \bar{x} b_{i}$ for $i \in\{0, \ldots, k\}$ and $\left(2 \beta+L \sum_{i=0}^{k} b_{i}\right)(\beta+$ $\left.\sum_{i=0}^{k} a_{i}+L \sum_{i=0}^{k} b_{i}\right)>\alpha \sum_{i=0}^{k} b_{i}>0$; or

(c) for some $i, j \in\{0, \ldots, k\} a_{i}>\bar{x} b_{i}, a_{j}<\bar{x} b_{j}$, and

$$
\frac{\alpha}{\bar{x}}+2 A_{S}-2 \bar{x} B_{S}<2 \beta+L \sum_{i=0}^{k} b_{i}
$$

where $\alpha \geq 0$,

then the positive equilibrium $\bar{x}$ of (1) is globally asymptotically stable on the interval $[0, \infty)$.

(2) If $\beta=0$ and either

(a) $a_{i}=\bar{x} b_{i}$ for all $i \in\{0, \ldots, k\}$;

(b) $a_{i} \leq \bar{x} b_{i}$ for $i \in\{0, \ldots, k\}$ and $0<\alpha<\bar{x} L \sum_{i=0}^{k} b_{i}$; or

(c) for some $i, j \in\{0, \ldots, k\} a_{i}>\bar{x} b_{i}, a_{j}<\bar{x} b_{j}$, and

$$
\frac{\alpha}{\bar{x}}+2 A_{S}-2 \bar{x} B_{S}<L \sum_{i=0}^{k} b_{i}
$$

where $\alpha \geq 0$,

then the positive equilibrium $\bar{x}$ of (1) is globally asymptotically stable on the interval $(0, \infty)$.

Proof. (1) Assume that $\beta>0$.

(a) Let $a_{i} \geq \bar{x} b_{i}$ for $i \in\{0, \ldots, k\}$. Since $\alpha=0$, then

$\sum_{i=0}^{k}\left|a_{i}-\bar{x} b_{i}\right|=\sum_{i=0}^{k} a_{i}-\bar{x} \sum_{i=0}^{k} b_{i}=\beta<\beta+L \sum_{i=0}^{k} b_{i}$

and the result follows from Theorem 7 .

(b) Let $a_{i} \leq \bar{x} b_{i}$ for $i \in\{0, \ldots, k\}$. By Lemma 8 with $L>0$ we get

$$
\sum_{i=0}^{k}\left|a_{i}-\bar{x} b_{i}\right|=\bar{x} \sum_{i=0}^{k} b_{i}-\sum_{i=0}^{k} a_{i}<\beta+L \sum_{i=0}^{k} b_{i}
$$


(c) Assume that for some $i, j \in\{0, \ldots, k\} a_{i}>\bar{x} b_{i}$ and $a_{j}<\bar{x} b_{j}$. Then

$$
\begin{aligned}
\sum_{i=0}^{k}\left|a_{i}-\bar{x} b_{i}\right| & =\bar{x} \sum_{i=0}^{k} b_{i}-\sum_{i=0}^{k} a_{i}+2 A_{S}-2 \bar{x} B_{S} \\
& =\frac{\alpha}{\bar{x}}-\beta+2 A_{S}-2 \bar{x} B_{S} \\
& <\beta+L \sum_{i=0}^{k} b_{i}
\end{aligned}
$$

and the result follows from Theorem 7.

(2) Assume that $\beta=0$.

(a) Let $a_{i}=\bar{x} b_{i}$ for all $i \in\{0, \ldots, k\}$. Then $\alpha=0$ and (1) becomes $x_{n+1}=\bar{x}$.

(b) Let $a_{i} \leq \bar{x} b_{i}$ for $i \in\{0, \ldots, k\}$. Then

$$
\sum_{i=0}^{k}\left|a_{i}-\bar{x} b_{i}\right|=\bar{x} \sum_{i=0}^{k} b_{i}-\sum_{i=0}^{k} a_{i}=\frac{\alpha}{\bar{x}}<L \sum_{i=0}^{k} b_{i}
$$

and the result follows from Theorem 7.

(c) Assume that for some $i, j \in\{0, \ldots, k\} a_{i}>\bar{x} b_{i}$ and $a_{j}<\bar{x} b_{j}$. Then

$$
\begin{aligned}
\sum_{i=0}^{k} \mid a_{i} & -\bar{x} b_{i} \mid \\
& =\bar{x} \sum_{i=0}^{k} b_{i}-\sum_{i=0}^{k} a_{i}+2 A_{S}-2 \bar{x} B_{S} \\
& =\frac{\alpha}{\bar{x}}+2 A_{S}-2 \bar{x} B_{S}<L \sum_{i=0}^{k} b_{i}
\end{aligned}
$$

and the result follows from Theorem 7.

By using Theorem 2 and similar methods as in the proof of Theorems 7, 9, and 11 we can obtain the conditions for the stability of the positive equilibrium.

Theorem 12. Let $\sum_{i=0}^{k} b_{i}>0$. Assume that one of the following holds:

(1) $\sum_{i=0}^{k}\left|a_{i}-\bar{x} b_{i}\right|=\beta$;

(2) there exist $L, N>0$ such that for every solution $\left\{x_{n}\right\}$ of (1) $x_{n} \geq L$ for all $n \geq N$ and $\sum_{i=0}^{k}\left|a_{i}-\bar{x} b_{i}\right|=\beta+$ $L \sum_{i=0}^{k} b_{i}$, where $\beta \geq 0$.

Then the positive equilibrium $\bar{x}$ of (1) is stable on the interval $[0, \infty)$.
Proof. (1) Observe that for $n \geq 0$

$$
\begin{aligned}
\sum_{i=0}^{k}\left|g_{i}\right| & =\sum_{i=0}^{k} \frac{\left|a_{i}-\bar{x} b_{i}\right|}{\beta+\sum_{i=0}^{k} b_{i} x_{n-i}} \\
& =\frac{\sum_{i=0}^{k}\left|a_{i}-\bar{x} b_{i}\right|}{\beta+\sum_{i=0}^{k} b_{i} x_{n-i}} \leq \frac{\sum_{i=0}^{k}\left|a_{i}-\bar{x} b_{i}\right|}{\beta}=1 .
\end{aligned}
$$

Thus the result follows from Theorem 2 .

(2) The result follows similarly as in Theorem 7 part (2).

Theorem 13. Let $\beta, \sum_{i=0}^{k} b_{i}>0$. Then the positive equilibrium $\bar{x}$ of $(1)$ is stable on the interval $[0, \infty)$ provided one of the following holds:

(1) $a_{i} \geq \bar{x} b_{i}$ for $i \in\{0, \ldots, k\}$ and $\alpha=0$;

(2) $a_{i} \leq \bar{x} b_{i}$ for $i \in\{0, \ldots, k\}$ and $2 \beta^{2}+2 \beta \sum_{i=0}^{k} a_{i}=$ $\alpha \sum_{i=0}^{k} b_{i}>0$;

(3) for some $i, j \in\{0, \ldots, k\}, a_{i}>\bar{x} b_{i}, a_{j}<\bar{x} b_{j}$, and $\alpha / \bar{x}+$ $2 A_{S}-2 \bar{x} B_{S}=2 \beta$ where $\alpha \geq 0$.

Theorem 14. Let $\sum_{i=0}^{k} b_{i}>0$ and let $L, N>0$ be such that for every solution $\left\{x_{n}\right\}$ of (1) $x_{n} \geq L$ for $n \geq N$.

(1) If $\beta>0$ and either

(a) $a_{i} \leq \bar{x} b_{i}$ for $i \in\{0, \ldots, k\}$ and $\left(2 \beta+L \sum_{i=0}^{k} b_{i}\right)(\beta+$ $\left.\sum_{i=0}^{k} a_{i}+L \sum_{i=0}^{k} b_{i}\right)=\alpha \sum_{i=0}^{k} b_{i}>0$ or

(b) for some $i, j \in\{0, \ldots, k\} a_{i}>\bar{x} b_{i}, a_{j}<\bar{x} b_{j}$, and

$$
\frac{\alpha}{\bar{x}}+2 A_{S}-2 \bar{x} B_{S}=2 \beta+L \sum_{i=0}^{k} b_{i}
$$

where $\alpha \geq 0$,

then the positive equilibrium $\bar{x}$ of (1) is stable on the interval $[0, \infty)$.

(2) If $\beta=0$ and either

(a) $a_{i} \leq \bar{x} b_{i}$ for $i \in\{0, \ldots, k\}$ and $0<\alpha=\bar{x} L \sum_{i=0}^{k} b_{i}$ or

(b) for some $i, j \in\{0, \ldots, k\} a_{i}>\bar{x} b_{i}, a_{j}<\bar{x} b_{j}$, and

$$
\frac{\alpha}{\bar{x}}+2 A_{S}-2 \bar{x} B_{S}=L \sum_{i=0}^{k} b_{i}
$$

where $\alpha \geq 0$,

then the positive equilibrium $\bar{x}$ of (1) is stable on the interval $(0, \infty)$.

When $\sum_{i=0}^{k}\left|a_{i}-\bar{x} b_{i}\right| \geq \beta$, the following results show that the positive equilibrium of (1) may be globally asymptotically stable on a subspace of the initial conditions. First we will need the following lemma. 
Lemma 15. Let $0<m \leq \bar{x}$, and for some $N \in\{0,1, \ldots\} x_{N-i} \geq$ $m, i=0, \ldots, k$. Suppose that

$$
x_{n+1}-\bar{x}=\sum_{i=0}^{k} h_{i}\left(x_{n-i}-\bar{x}\right), \quad n=0,1, \ldots,
$$

where the nonnegative functions $h_{i}:[0, \infty)^{k+1} \rightarrow[0, \infty)$. Assume that, for this $N, \sum_{i=0}^{k} h_{i}<1$. Then $x_{N+1} \geq m$.

Proof. Let $K \in \mathbb{R}$. Then (69) has the generalized identity

$$
\begin{array}{r}
x_{n+1}-\bar{x}-K \sum_{i=0}^{k} h_{i}=\sum_{i=0}^{k} h_{i}\left[\left(x_{n-i}-\bar{x}\right)-K\right], \\
n=0,1, \ldots
\end{array}
$$

Choose $N \in\{0,1, \ldots\}$. First, suppose that $x_{N-i} \geq \bar{x}$ for $i=0, \ldots, k$. Then by $(69)$

$$
x_{N+1}-\bar{x}=h_{0}\left(x_{N}-\bar{x}\right)+\cdots+h_{k}\left(x_{N-k}-\bar{x}\right) \geq 0 .
$$

Thus $x_{N+1} \geq m$.

Second, suppose that $m \leq x_{N-i}<\bar{x}$ for some $i \epsilon$ $\{0, \ldots, k\}$. Then $m<\bar{x}$. Let $K=m-\bar{x}$. Then

$$
x_{N+1}-\bar{x}-(m-\bar{x}) \sum_{i=0}^{k} h_{i}=\sum_{i=0}^{k} h_{i}\left(x_{N-i}-m\right) \geq 0 .
$$

By assumption $\sum_{i=0}^{k} h_{i}<1$ for this $N$ and so

$$
x_{N+1}-\bar{x} \geq(m-\bar{x}) \sum_{i=0}^{k} h_{i}>m-\bar{x} .
$$

Thus $x_{N+1}>m$.

Theorem 16. Let $\sum_{i=0}^{k} b_{i}>0$ and $m=\min \left\{\bar{x}, x_{0}, \ldots, x_{-k}\right\}$. Assume that $0 \leq \sum_{i=0}^{k}\left|a_{i}-\bar{x} b_{i}\right|-\beta<m \sum_{i=0}^{k} b_{i}$. Then the positive equilibrium of (1) is globally asymptotically stable on the interval $\left(\left(\sum_{i=0}^{k}\left|a_{i}-\bar{x} b_{i}\right|-\beta\right) / \sum_{i=0}^{k} b_{i}, \infty\right)$.

Proof. Clearly $0<m$. Since $m \leq x_{-i}$ for $i=0, \ldots, k$, then $m \sum_{i=0}^{k} b_{i} \leq \sum_{i=0}^{k} b_{i} x_{-i}$ and so

$$
\sum_{i=0}^{k}\left|g_{i}\right|=\sum_{i=0}^{k} \frac{\left|a_{i}-\bar{x} b_{i}\right|}{\beta+\sum_{i=0}^{k} b_{i} x_{-i}} \leq \frac{\sum_{i=0}^{k}\left|a_{i}-\bar{x} b_{i}\right|}{\beta+m \sum_{i=0}^{k} b_{i}}<1
$$

for $n=0$. Then by Lemma 15 with $h_{i}=\left|g_{i}\right|$ for $i \in\{0, \ldots, k\}$ and $N=0$ we get that $x_{1} \geq m$. Hence $m \leq x_{1}, \ldots, x_{-k}$. Thus

$$
\sum_{i=0}^{k}\left|g_{i}\right|=\sum_{i=0}^{k} \frac{\left|a_{i}-\bar{x} b_{i}\right|}{\beta+\sum_{i=0}^{k} b_{i} x_{1-i}} \leq \frac{\sum_{i=0}^{k}\left|a_{i}-\bar{x} b_{i}\right|}{\beta+m \sum_{i=0}^{k} b_{i}}<1
$$

for $n=1$. Now applying Lemma 15 again with $h_{i}=\left|g_{i}\right|$ for $i \in\{0, \ldots, k\}$ and $N=1$ we get that $x_{2} \geq m$ and so $m \leq$ $x_{2}, \ldots, x_{-k}$. Thus

$$
\sum_{i=0}^{k}\left|g_{i}\right|=\sum_{i=0}^{k} \frac{\left|a_{i}-\bar{x} b_{i}\right|}{\beta+\sum_{i=0}^{k} b_{i} x_{2-i}} \leq \frac{\sum_{i=0}^{k}\left|a_{i}-\bar{x} b_{i}\right|}{\beta+m \sum_{i=0}^{k} b_{i}}<1
$$

for $n=2$. Hence by induction we get that

$$
\sum_{i=0}^{k}\left|g_{i}\right|=\sum_{i=0}^{k} \frac{\left|a_{i}-\bar{x} b_{i}\right|}{\beta+\sum_{i=0}^{k} b_{i} x_{n-i}} \leq \frac{\sum_{i=0}^{k}\left|a_{i}-\bar{x} b_{i}\right|}{\beta+m \sum_{i=0}^{k} b_{i}}<1
$$

for $n=0,1, \ldots$. Therefore applying Theorem 1 to $(24)$ we have that

$$
\lim _{n \rightarrow \infty} y_{n}=0 \text { and so } \lim _{n \rightarrow \infty} x_{n}=\bar{x}
$$

and by applying Theorem 2 to (24) $\bar{x}$ is stable.

We now apply Theorem 16 to various cases of (1).

Theorem 17. Let $\sum_{i=0}^{k} b_{i}>0$ and $m=\min \left\{\bar{x}, x_{0}, \ldots, x_{-k}\right\}$. Then the positive equilibrium $\bar{x}$ of (1) is globally asymptotically stable on the interval $\left(\left(\sum_{i=0}^{k}\left|a_{i}-\bar{x} b_{i}\right|-\beta\right) / \sum_{i=0}^{k} b_{i}, \infty\right) \subset[0, \infty)$ provided one of the following holds:

(1) $a_{i}=\bar{x} b_{i}$ for all $i \in\{0, \ldots, k\}$

(2) $a_{i} \geq \bar{x} b_{i}$ for $i \in\{0, \ldots, k\}, \alpha=0, \beta>0$, and $m>0$;

(3) $a_{i} \leq \bar{x} b_{i}$ for $i \in\{0, \ldots, k\}$ and either

(a) $\beta>0,2 \beta^{2}+2 \beta \sum_{i=0}^{k} a_{i} \leq \alpha \sum_{i=0}^{k} b_{i}$ and $\left(\bar{x} \sum_{i=0}^{k} b_{i}-\sum_{i=0}^{k} a_{i}-\beta\right) / \sum_{i=0}^{k} b_{i}<m$ or

(b) $\beta=0$ and $0<\alpha / \bar{x} \sum_{i=0}^{k} b_{i}<m$;

(4) for some $i, j \in\{0, \ldots, k\} a_{i}>\bar{x} b_{i}, a_{j}<\bar{x} b_{j}$, and $0 \leq$ $\alpha / \bar{x}-2 \beta+2 A_{S}-2 \bar{x} B_{S}<m \sum_{i=0}^{k} b_{i}$.

Proof. (1) If $\alpha, \beta>0$, then Theorem 9 part (1) applies. If $\alpha=$ $\beta=0$, (1) becomes

$$
x_{n+1}=\frac{\sum_{i=0}^{k} a_{i} x_{n-i}}{\sum_{i=0}^{k} b_{i} x_{n-i}}=\frac{\bar{x} \sum_{i=0}^{k} b_{i} x_{n-i}}{\sum_{i=0}^{k} b_{i} x_{n-i}}=\bar{x} .
$$

(2) In this case we have $\left|a_{i}-\bar{x} b_{i}\right|=a_{i}-\bar{x} b_{i}$ for $i \in\{0, \ldots, k\}$ and since $\alpha=0$, then $\beta=\sum_{i=0}^{k} a_{i}-\bar{x} \sum_{i=0}^{k} b_{i}=\sum_{i=0}^{k}\left|a_{i}-\bar{x} b_{i}\right|$. Thus $0=\left(\sum_{i=0}^{k}\left|a_{i}-\bar{x} b_{i}\right|-\beta\right) / \sum_{i=0}^{k} b_{i}<m$ and the result follows from Theorem 16.

(3) In this case we have $\left|a_{i}-\bar{x} b_{i}\right|=\bar{x} b_{i}-a_{i}$ for $i \in\{0, \ldots, k\}$.

(a) Assume that $\beta>0,2 \beta^{2}+2 \beta \sum_{i=0}^{k} a_{i} \leq \alpha \sum_{i=0}^{k} b_{i}$ and $\left(\bar{x} \sum_{i=0}^{k} b_{i}-\sum_{i=0}^{k} a_{i}-\beta\right) / \sum_{i=0}^{k} b_{i}<m$. By Lemma 8 with $L=0, \beta+\sum_{i=0}^{k} a_{i} \leq \bar{x} \sum_{i=0}^{k} b_{i}$. Thus

$$
0 \leq \frac{\sum_{i=0}^{k}\left|a_{i}-\bar{x} b_{i}\right|-\beta}{\sum_{i=0}^{k} b_{i}}<m
$$

and so the result follows from Theorem 16.

(b) Assume that $\beta=0$ and $0<\alpha / \bar{x} \sum_{i=0}^{k} b_{i}<m$. Then

$$
\frac{\alpha}{\bar{x}}=\bar{x} \sum_{i=0}^{k} b_{i}-\sum_{i=0}^{k} a_{i}=\sum_{i=0}^{k}\left|a_{i}-\bar{x} b_{i}\right| .
$$


Thus $0<\sum_{i=0}^{k}\left|a_{i}-\bar{x} b_{i}\right| / \sum_{i=0}^{k} b_{i}<m$ and so the result follows from Theorem 16 .

(4) In this case from the equilibrium equation we get that

$$
\begin{aligned}
\sum_{i=0}^{k} \mid a_{i} & -\bar{x} b_{i} \mid-\beta \\
& =\bar{x} \sum_{i=0}^{k} b_{i}-\sum_{i=0}^{k} a_{i}+2 A_{S}-2 \bar{x} B_{S}-\beta \\
& =\frac{\alpha}{\bar{x}}-\beta+2 A_{S}-2 \bar{x} B_{S}-\beta .
\end{aligned}
$$

Suppose that $\beta \geq 0$ and $\alpha \geq 0$. Then

$$
0 \leq \frac{\sum_{i=0}^{k}\left|a_{i}-\bar{x} b_{i}\right|-\beta}{\sum_{i=0}^{k} b_{i}}<m
$$

and so the result follows from Theorem 16.

We illustrate our results with some examples.

Example 18. Equation

$$
x_{n+1}=\frac{\alpha+\beta x_{n}+\gamma x_{n-1}+\delta x_{n-2}}{A+B x_{n}+C x_{n-1}}, \quad n=0,1, \ldots,
$$

with nonnegative initial conditions and positive coefficients was considered in [4], and it was proved that the condition $A \geq \beta+\gamma+\delta$ implies global asymptotic stability. By Theorem 7 the condition

$$
\begin{array}{r}
|\beta-B \bar{x}|+|\gamma-C \bar{x}|+\delta<A+(B+C) L, \\
L=\frac{\min \{\alpha, \beta, \gamma, \delta\}}{\max \{A, B, C\}},
\end{array}
$$

implies global asymptotic stability of the unique positive equilibrium $\bar{x}$. If $\bar{x} \leq \beta / B, \bar{x} \leq \gamma / C$, then the sufficient condition for global asymptotic stability of the unique positive equilibrium $\bar{x}$ of (84) becomes

$$
\beta+\gamma+\delta<A+(B+C)(L+\bar{x}) .
$$

Example 19. Equation

$$
x_{n+1}=\frac{\alpha+x_{n-1}}{A+B x_{n}+x_{n-1}+D x_{n-2}}, \quad n=0,1, \ldots,
$$

with nonnegative initial conditions and positive coefficients was considered in [4], and it was proved that the condition $A \geq 1$ implies global asymptotic stability. Theorem 7 implies that, for the condition

$$
|1-\bar{x}|<A+(B+D)(L-\bar{x})+L,
$$

where

$$
L=\frac{\min \{\alpha, 1\}}{\max \{A+(B+D) U, 1\}}, \quad U=\frac{\max \{\alpha, 1\}}{\min \{A, B, D, 1\}},
$$

the positive equilibrium $\bar{x}$ is globally asymptotically stable. If $\bar{x} \leq 1$ then the sufficient condition for global asymptotic stability of the unique positive equilibrium $\bar{x}$ of (87) becomes

$$
1<\bar{x}+A+(B+D)(L-\bar{x})+L \text {. }
$$

Example 20. Equation

$$
x_{n+1}=\frac{\beta x_{n}+\gamma x_{n-1}+\delta x_{n-2}}{B x_{n}+C x_{n-1}+D x_{n-2}}, \quad n=0,1, \ldots,
$$

with nonnegative initial conditions and positive coefficients was considered in $[4,10]$. In view of Theorem $7 \bar{x}$ is globally asymptotically stable if the condition

$$
|\beta-B \bar{x}|+|\gamma-C \bar{x}|+|\delta-D \bar{x}|<(B+C+D) L,
$$

where

$$
L=\frac{\min \{\beta, \gamma, \delta\}}{\max \{B, C, D\}}, \quad \bar{x}=\frac{\beta+\gamma+\delta}{B+C+D},
$$

is satisfied. For instance if

$$
\frac{\beta}{B}=\frac{\gamma}{C}<\frac{\delta}{D}
$$

then it is clear that $\beta / B=\gamma / C<\bar{x}<\delta / D$ and condition (92) becomes

$$
\begin{gathered}
-(\beta-B \bar{x})-C(\beta-B \bar{x})+\delta-D \bar{x}<(B+C+D) L \\
\text { or }((C+1) B-D) \bar{x}<(B+C+D) L+(C+1) \beta-\delta . \\
\text { If } \\
\frac{\beta}{B}<\frac{\gamma}{C}<\frac{\delta}{D},
\end{gathered}
$$

then it is clear that $\beta / B<\bar{x}<\delta / D$ and condition (92) becomes

$$
-(\beta-B \bar{x})+|\beta-B \bar{x}|+\delta-D \bar{x}<(B+C+D) L
$$

or $|\beta-B \bar{x}|<\beta-\delta+(D-B) \bar{x}+(B+C+D) L$.

Note that, by Theorem 7, condition (92) also implies that the positive equilibrium $\bar{x}$ is globally asymptotically stable even for the special cases of (91) where exactly one of the coefficients $B, C$, or $D$ is zero. These three cases were considered in [4] but no global stability or attractivity results were presented.

As it was shown in $[10,11](91)$ has a minimal period-two solution for some values of the parameters.

Example 21. Equation

$$
x_{n+1}=\frac{\alpha+\beta x_{n}+\gamma x_{n-1}+\delta x_{n-2}}{B x_{n}+C x_{n-1}}, \quad n=0,1, \ldots,
$$

with nonnegative initial conditions and positive coefficients was considered in [4], but no global stability or attractivity results were presented. In view of Theorem 7 , the positive equilibrium $\bar{x}$ is globally asymptotically stable if the condition

$$
|\beta-B \bar{x}|+|\gamma-C \bar{x}|+\delta<(B+C) L,
$$

where

$$
L=\frac{\min \{\alpha, \beta, \gamma, \delta\}}{\max \{B, C\}},
$$

is satisfied. 


\section{Conflict of Interests}

The authors declare that there is no conflict of interests regarding the publication of this paper.

\section{References}

[1] M. R. S. Kulenović and G. Ladas, Dynamics of Second Order Rational Difference Equations, with Open Problems and Conjectures, Chapman \& Hall/CRC, 2001.

[2] E. Camouzis and G. Ladas, Dynamics of Third Order Rational Difference Equations, with Open Problems and Conjectures, Chapman \& Hall/CRC, 2007.

[3] D. Burgić, S. Kalabušić, and M. R. S. Kulenović, "Period-two trichotomies of a difference equation of order higher than two," Sarajevo Journal of Mathematics, vol. 4, no. 16, pp. 73-90, 2008.

[4] E. Camouzis and G. Ladas, "When does local asymptotic stability imply global attractivity in rational equations?" Journal of Difference Equations and Applications, vol. 12, no. 8, pp. 863885, 2006.

[5] F. J. Palladino, "On the characterization of rational difference equations," Journal of Difference Equations and Applications, vol. 15, no. 3, pp. 253-260, 2009.

[6] F. J. Palladino, "On periodic trichotomies," Journal of Difference Equations and Applications, vol. 15, no. 6, pp. 605-620, 2009.

[7] S. Kalabušić, M. R. S. Kulenović, and C. B. Overdeep, "Dynamics of the recursive sequence $x_{n+1}=\left(\beta x_{n-l}+\delta x_{n-k}\right) /\left(B x_{n-l}+\right.$ $\left.D x_{n-k}\right)$," Journal of Difference Equations and Applications, vol. 10, pp. 915-928, 2004.

[8] E. Bedford and K. Kim, "Periodicities in linear fractional recurrences: degree growth of birational surface maps," Michigan Mathematical Journal, vol. 54, no. 3, pp. 647-671, 2006.

[9] E. Bedford and K. Kim, "Dynamics of rational surface automorphisms: linear fractional recurrences," Journal of Geometric Analysis, vol. 19, no. 3, pp. 553-583, 2009.

[10] E. M. Elabbasy, H. El-Metwally, and E. M. Elsayed, "Global attractivity and periodic character of a fractional difference equation of order three," The Yokohama Mathematical Journal, vol. 53, no. 2, pp. 89-100, 2007.

[11] E. M. Elabbasy, H. El-Metwally, and E. M. Elsayed, "On the difference equation $x_{n+1}=\left(a_{0} x_{n}+a_{1} x_{n 1}+\ldots+a_{k} x_{n-k}\right) /\left(b_{0} x_{n}+\right.$ $\left.b_{1} x_{n 1}+\ldots+b_{k} x_{n-k}\right)$," Mathematica Bohemica, vol. 133, pp. 133147, 2008.

[12] J. Bektešević, M. R. S. Kulenović, and E. Pilav, "Global dynamics of quadratic second order difference equation in the first quadrant," Applied Mathematics and Computation, vol. 227, pp. 50-65, 2014.

[13] V. L. Kocic and G. Ladas, Global Behavior of Nonlinear Difference Equations of Higher Order with Applications, Kluwer Academic, Dodrecht, The Netherlands, 1993.

[14] M. R. S. Kulenović and O. Merino, Discrete Dynamical Systems and Difference Equations with Mathematica, Chapman \& Hall/CRC, Boca Raton, Fla, USA, 2002.

[15] M. R. S. Kulenović and O. Merino, "A global attractivity result for maps with invariant boxes," Discrete and Continuous Dynamical Systems B, vol. 6, no. 1, pp. 97-110, 2006.

[16] R. D. Nussbaum, "Global stability, two conjectures and Maple," Nonlinear Analysis: Theory, Methods \& Applications, vol. 66, no. 5, pp. 1064-1090, 2007.
[17] H. Sedaghat, Nonlinear Difference Equations, Theory with Applications to Social Science Models, vol. 15 of Mathematical Modelling: Theory and Applications, Kluwer Academic, Dodrecht, The Netherlands, 2003.

[18] E. J. Janowski and M. R. S. Kulenović, "Attractivity and global stability for linearizable difference equations," Computers and Mathematics with Applications, vol. 57, no. 9, pp. 1592-1607, 2009.

[19] E. Camouzis, G. Ladas, F. Palladino, and E. P. Quinn, "On the boundedness character of rational equations, part 1," Journal of Difference Equations and Applications, vol. 12, no. 5, pp. 503-523, 2006.

[20] M. R. S. Kulenović and M. Mehuljić, "Global behavior of some rational second order difference equations," International Journal of Difference Equations, vol. 7, pp. 151-160, 2012. 


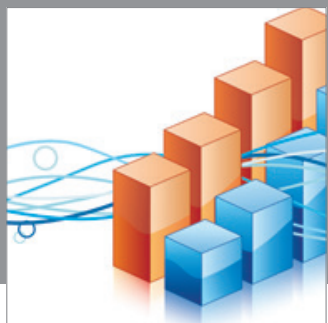

Advances in

Operations Research

mansans

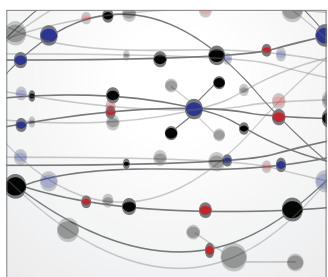

The Scientific World Journal
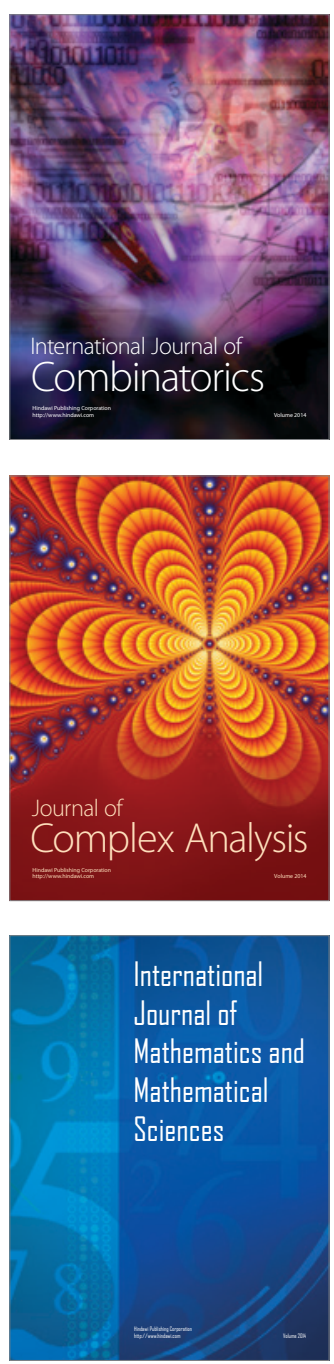
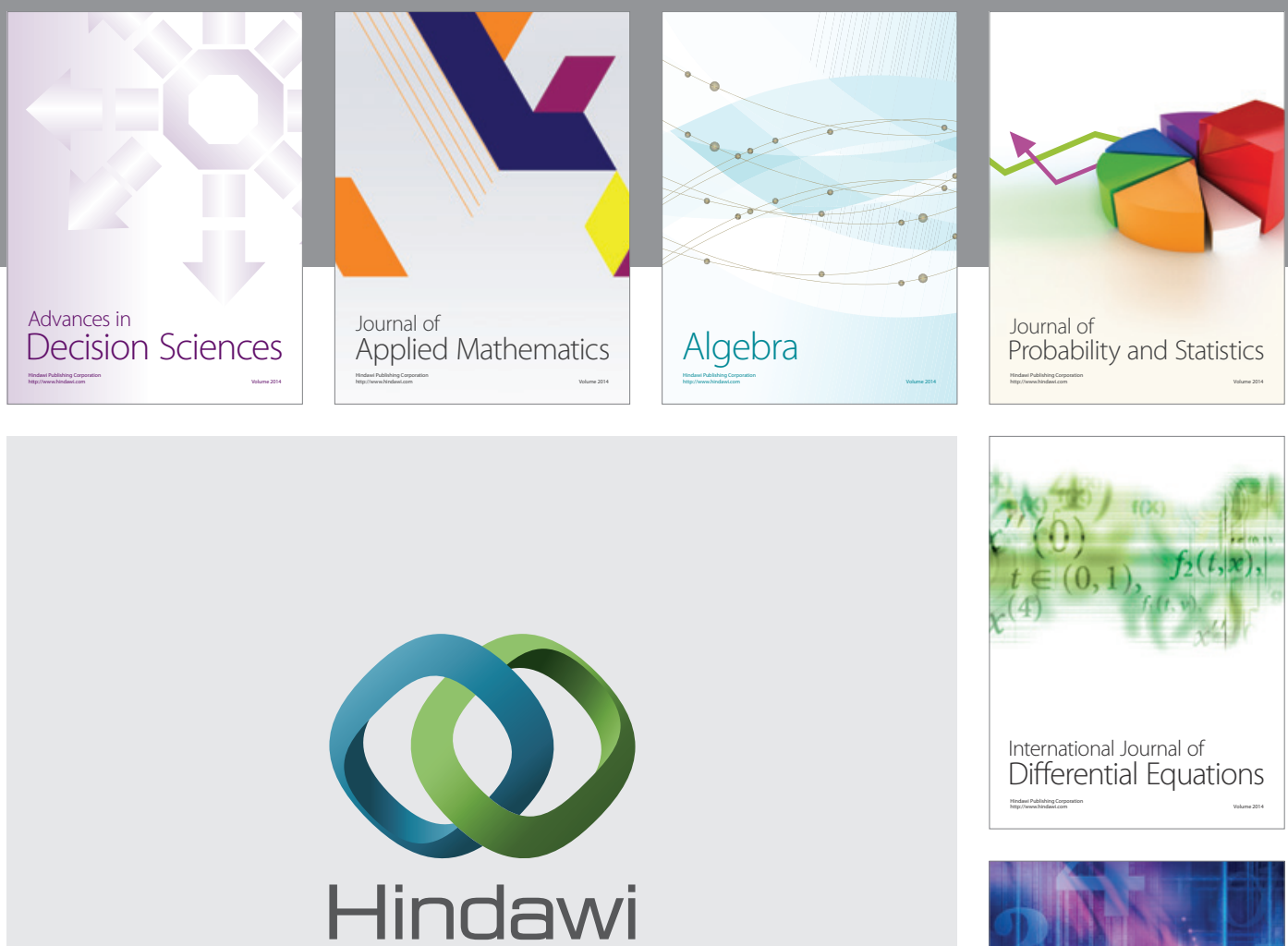

Submit your manuscripts at http://www.hindawi.com
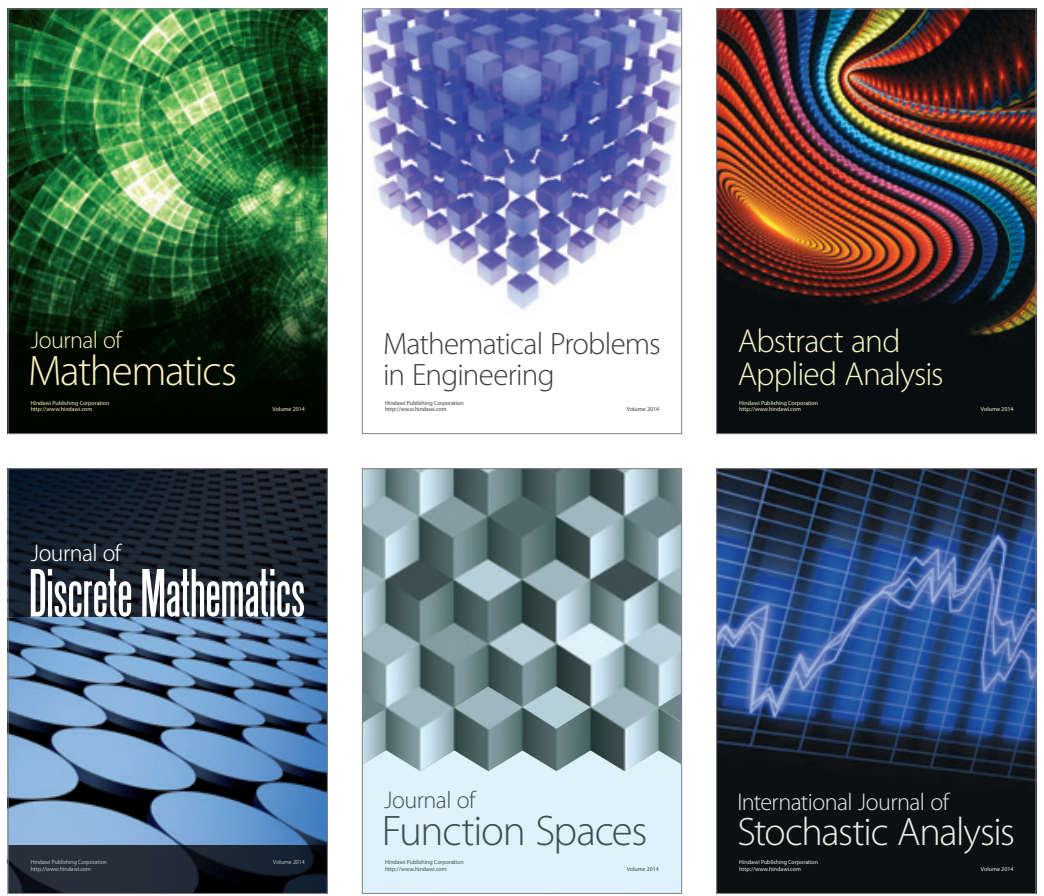

Journal of

Function Spaces

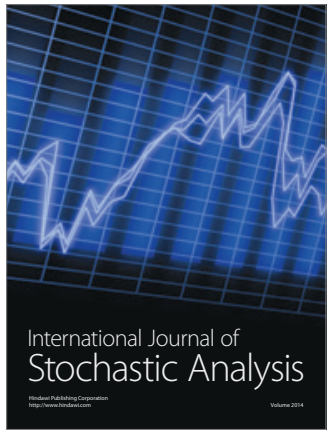

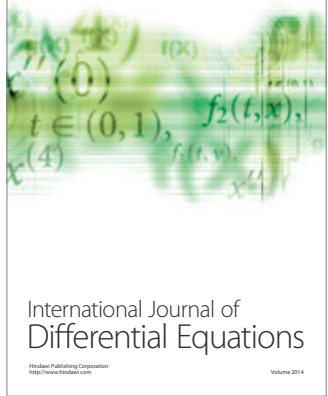
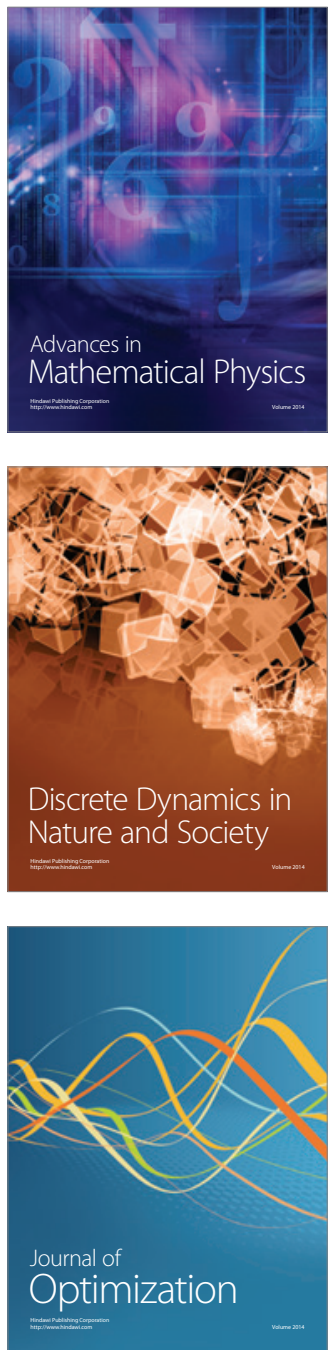